\title{
Christoph Schönberger, Der „German Approach“
}

\section{Philipp Siegert}

\section{(2) OpenEdition \\ Journals}

Édition électronique

URL : http://journals.openedition.org/ifha/8668

DOI : $10.4000 /$ ifha. 8668

ISSN : 2198-8943

Éditeur

IFRA - Institut franco-allemand (sciences historiques et sociales)

\section{Référence électronique}

Philipp Siegert, «Christoph Schönberger, Der „German Approach“", Revue de I'IFHA [En ligne], Date de recension, mis en ligne le 01 février 2017, consulté le 24 septembre 2020. URL : http:// journals.openedition.org/ifha/8668; DOI : https://doi.org/10.4000/ifha.8668

Ce document a été généré automatiquement le 24 septembre 2020.

(CIFHA 


\title{
Christoph Schönberger, Der „German Approach"
}

\author{
Philipp Siegert
}

\section{RÉFÉRENCE}

Christoph Schönberger, Der „German Approach“, Tübingen: Mohr Siebeck, 2015

(Fundamenta Juris Publici 4), 121 p., $16 €$ 
Issu d'un colloque de la section «Fondements du droit public» ( "Grundlagen des öffentlichen Rechts») qui s'est tenu lors de la rencontre annuelle de la très prestigieuse Vereinigung der deutschen Staatsrechtslehrer en 2014, ce livre regroupe une conférence de Christoph Schönberger, le commentaire d'Atsushi Takada (Japon) et un essai d'András Jakab (Hongrie).

L'auteur principal se penche sur l'évolution atypique du droit public allemand, et en particulier de sa doctrine (la Staatsrechtslehre), en retraçant son développement depuis l'époque romantique jusqu'à nos jours. La langue est d'une clarté exceptionnelle compte tenu de la complexité du sujet. Le terme de "German Approach » désigne une façon de penser, une manière de procéder dans la science du droit public qui serait propre à l'Allemagne. L'antériorité de la Staatsrechtslehre par rapport à l'État lui-même serait ainsi au fondement de la spécificité de la German Approach: "le 'droit scientifique' se présente comme une pratique poétique, qui en partie remplace et en partie transcende le droit positif» (p. 2). Cette science revêt en quelque sorte le rôle de précurseur d'un État allemand (unifié). Au XIX siècle, la science du droit aurait ainsi «pris la place d'institutions fragmentées, particularistes ou tout à fait absentes »(p.20). Une autre caractéristique serait la tendance à travailler de manière comparatiste, le droit français jouant un rôle particulier: de part et d'autre du Rhin, on retrouve en effet la «tradition de droit européen continental, fondée sur le droit romain, l'État administré (Verwaltungsstaat) et la carrière bureaucratique de juristes »(p.8), ce qui diffère de la situation anglosaxonne. Le choix de la France comme point de comparaison pour cette étude rend l'analyse particulièrement intéressante pour un lecteur français. Le regard comparatif franco-allemand permet, entre autres, d'évoquer les conséquences de l'existence (selon le modèle unitariste) ou de l'absence (selon le modèle fédéraliste) de cours suprêmes (p. 9, 42), le développement du droit par la jurisprudence (en France) ou par la science (en Allemagne) (p. 23), ou encore la place différente occupée par la doctrine (Dogmatik) dans les deux pays (p. 39).

Un autre grand atout du texte est la richesse des notes de bas de page renvoyant à la littérature secondaire (cela vaut aussi pour la contribution d'A. Jakab). Le lecteur qui souhaite s'informer davantage sur le sujet y trouvera de précieuses indications. Par ailleurs, la structure de la conférence permet au lecteur de s'y retrouver, qu'il soit ou non familier de la Staatsrechtslehre. Partant de la «science allemande du droit comme projet romantique» (chap.1), C. Schönberger retrace son évolution en prenant en compte ses aspects épistémologique ("La comparaison comme outil de recherche", chap. 2), historique («L'absence d'État [unifié] comme espace d'évolution pour la 
German Approach », chap. 3, et " Après la catastrophe » [du nazisme], chap. 4), théorique («La fin de la tradition des projets conceptuels», chap.5), pour terminer par une esquisse de l'état actuel de la German Approach ("Depuis la réunification: malaise», chap. 6, et «La German Approach a-t-elle encore une chance?», chap. 7). Toutes les réflexions de l'auteur entendent expliquer et contextualiser cette tradition de pensée juridique allemande sur le plan historique et théorique, pour ensuite en tirer des conclusions sur son applicabilité dans les conditions contemporaines. Ce double objectif est parfaitement atteint.

L'auteur souligne plusieurs particularités de la German Approach et de la Staatsrechtslehre au XIX ${ }^{\mathrm{e}}$ siècle, en particulier sa "forte dimension prospective" (p. 21). Il retrace son évolution jusqu'en 1900, avant de revenir sur ladite "prospectivité » philosophique pendant la République de Weimar, où le manque de jurisprudence unitaire au niveau fédéral aurait laissé beaucoup d'espace à la German Approach et à ses " grands projets en science du droit » (p. 26). Suit l'échec de cette approche sous les nationaux-socialistes, phase dans laquelle "la tradition du droit scientifique des professeurs s'est transformée en und idée de science du droit public détachée aussi bien des notions caduques du droit public et administratif libéral que de leur contrôle par la justice» (p. 26). Conséquence : le discrédit et une rupture profonde empêchèrent cette science de participer activement à la reconstruction étatique de l'après-guerre; elle fut remplacée par la jurisprudence des cours fédérales, dorénavant renforcées. À partir des années 1960, la RFA se caractérise par une reconfiguration de la coopération entre science et jurisprudence : ce n'est plus la cour qui cherche à se légitimer en faisant référence à la science (pratique du Reichsgericht), mais l'inverse. Ceci n'est qu'un exemple parmi de nombreux autres présentés par C. Schönberger.

C'est surtout dans le chapitre final que l'auteur établit des liens avec la situation actuelle en Europe et avec le droit européen. Il constate d'abord qu'une science qui planerait au-dessus du droit lui-même et qui serait l'une de ses sources (donc supérieure au droit positif) "n'a plus de place au sein d'un droit positif créé démocratiquement » (p.47). En revanche, la qualité prospective qu'aurait eu la German Approach, du moins pendant la première moitié du XIX ${ }^{e}$ siècle, retrouverait un intérêt : "Européanisation et internationalisation confrontent les juristes d'aujourd'hui comme autrefois la Staatsrechtswissenschaft du XIX ${ }^{e}$ siècle en Allemagne - à un ensemble de textes juridiques et de décisions jurisprudentielles extrêmement hétérogène qui demande une systématisation scientifique étoffée» (p.50). Il y aurait donc dans l'histoire de la German Approach des aspects instructifs pour les temps présents. Qu'on partage cet avis ou non (on le verra chez A. Jakab), l'étude de C. Schönberger est riche d'enseignements.

Tout aussi pertinentes, les contributions d'A. Takada (p. 55-73) et d'A. Jakab (p. 75-121) apportent un regard extérieur (européen et extra-européen) au modus teutonicus. Fondé sur l'expérience japonaise, le texte d'A. Takada se concentre non seulement sur la possibilité d'appropriation de certains éléments du droit allemand en dehors de l'Allemagne, mais aussi, plus intéressant encore, sur les transformations de la Staatsrechtslehre et de sa fonction, en proposant des pistes de réflexion sur son évolution future (p.65-72). On y trouve notamment l'idée d'une différenciation disciplinaire aussi bien verticale qu'horizontale.

La contribution d'A. Jakab est plus programmatique. En se livrant à une étude historique et théorique, il estime que la Staatslehre (au sens d'une théorie générale de 
l'État comme l'a conçue notamment Georg Jellinek) n'offre « aucun cadre conceptuel pertinent pour le discours constitutionnel européen, car elle ne répond pas aux exigences des notions modernes de démocratie et de constitution » (p. 77). Au cours de son exposé, A. Jakab met en exergue les différentes compréhensions de «l'État » en Allemagne et dans d'autres pays européens qui expliqueraient en partie la particularité de la German Approach et de la Staatslehre par rapport à d'autres traditions juridiques et scientifiques (autrichienne ou britannique, par exemple). D'après lui, la Staatslehre serait une théorie constitutionnelle reposant sur une conception de l'État comme « entité antérieure au droit » (p. 82, 91), et c'est à partir de ce constat qu'il développe sa réponse quant à l'utilité actuelle de la Staatslehre. L'essai se conclut sur une analyse qui vaut à elle seule la peine d'être lue - de l'antagonisme entre l'État (comme « entité pré-légale ») et la constitution sous l'état d'urgence (p. 106-119). Il se distancie alors du texte de $C$. Schönberger, dans que son intérêt ne s'en trouve aucunement diminué.

Ces trois contributions livrent des réflexions historiques, épistémologiques et théoriques d'une grande pertinence. Très lisibles, elles intéresseront un public bien plus large que celui des seuls juristes.

INDEX

Index chronologique : Époque contemporaine

Thèmes : Histoire du droit, Histoire des sciences, Histoire des idées 